\title{
Diseño universal para el aprendizaje de las matemáticas en la formación inicial del profesorado
}

\author{
Ximena D. Gutiérrez-Saldivia*, Cecilia M. Barría y Carmen P. Tapia \\ Universidad Católica de Temuco, Facultad de Educación, Dpto. de Infancia y Educación Básica, Carrera Pedagogía en \\ Educación Diferencial, Rudecindo Ortega 02950, Temuco, Chile. (correo-e: xgutierrez.saldivia@gmail.com; \\ cbarria@uct.cl; ctapia@uct.cl)
}

* Autor a quien debe ser dirigida la correspondencia

Recibido Mar. 26, 2020; Aceptado May. 28, 2020; Versión final Jul. 30, 2020, Publicado Dic. 2020

\begin{abstract}
Resumen
El objetivo de este estudio es identificar las estrategias del diseño universal para el aprendizaje (DUA) más valoradas por los estudiantes del curso "herramientas de acceso para el aprendizaje del lenguaje y las matemáticas I", así como describir los facilitadores y obstaculizadores para el aprendizaje en la implementación del DUA. El diseño es de investigación-acción, utilizándose una escala de valoración y un cuestionario abierto. Los participantes son 80 estudiantes. Los resultados indican que la implementación del DUA en el curso facilitó la comprensión de los contenidos, propició la participación y acercó a los estudiantes a su futuro profesional. Los estudiantes destacan como facilitadores las estrategias de aprendizaje, recursos materiales y características personales y profesionales de las profesoras; mientras que los obstaculizadores son los periodos de clases, el tamaño y la acústica de las salas. Se concluye que el DUA genera mayores oportunidades de aprendizaje, ya que ofrece múltiples estrategias para que todos los estudiantes desarrollen competencias profesionales.
\end{abstract}

Palabras clave: diseño universal para el aprendizaje; educación especial; formación inicial docente; matemáticas

\section{Universal design for learning mathematics in the initial stages of teacher training}

\begin{abstract}
This research study aims to identify the most valued universal design for learning (UDL) strategies by students taking the course "access tools for learning language and mathematics I." This study also aims to describe learning facilitators and obstacles in UDL implementation. The methodological design is action-research, using a rating scale and open-ended questions. The participants involved are 80 students. The results show that UDL implementation facilitated the students' course content understanding, encouraged active participation, and served as an introduction to professional prospects. The learning facilitators highlighted by students include the learning strategies, the resources available, and the teachers' personal and professional characteristics. The learning obstacles include class length and classroom size and acoustics. It is concluded that UDLs improve learning opportunities since they offer multiple strategies for professional skill development to all students.
\end{abstract}




\section{INTRODUCCIÓN}

En Chile, durante los últimos treinta años, la educación superior ha experimentado procesos de expansión, lo que ha permitido que más de un millón de jóvenes ingresen a las universidades (Organización de las Naciones Unidades para la Educación, la Ciencia y la Cultura UNESCO, 2015). Actualmente, la matrícula total de pregrado corresponde a 1.180.181 (Consejo Nacional de Educación, 2020), lo que demuestra un incremento de estudiantes en este nivel educativo. Dicho aumento se debe a la implementación de políticas educativas neoliberales, creándose diversos programas de acceso inclusivo que promueve el Ministerio de Educación MINEDUC para estudiantes de contextos vulnerables con rendimiento académico destacado. Sin embargo, estas políticas han sido incapaces de disminuir las desigualdades sociales y económicas, continuando con la promoción de un sistema educativo privatizante (Undurraga, 2015), que ha excluido desde la dictadura militar a profesores y estudiantes en la formulación de las políticas educativas (Inzunza et al., 2019). Por lo tanto, la expansión de la matrícula en la educación superior no solo significa un mayor número de estudiantes, sino también una población más heterogénea en cuanto a edades, intereses, origen cultural, experiencias de vida, habilidades y preferencias de aprendizajes (Dean et al., 2016), y que demanda de docentes y estudiantes críticos y propositivos para mejorar la calidad de la educación.

Los antecedentes presentados anteriormente desafían a las universidades a ofrecer entornos de aprendizajes diversificados e inclusivos donde se reconozcan las diferencias de todos los estudiantes y las limitaciones del sistema educativo chileno. Ello implica en la educación superior y especialmente en los docentes emprender cambios en la enseñanza para avanzar hacia prácticas donde el profesor sea un gestor, guía y facilitador del aprendizaje. Debido a esto, es que surge el interés de implementar el diseño universal para el aprendizaje (DUA) en el curso herramientas de acceso para el aprendizaje del lenguaje y las matemáticas I, que se sitúa en el sexto semestre del itinerario formativo de la carrera de pedagogía en educación diferencial, de una universidad del sur de Chile. Además, es fundamental aportar a la construcción de conocimiento sobre el DUA en la formación inicial docente, ya que, el MINEDUC en el 2014 estableció el DUA como un saber disciplinar en los estándares orientadores para carreras de educación especial. En este contexto, se justifica la realización de este estudio, ya que en nuestro país existe con un desarrollo incipiente de investigaciones (Sánchez-Gómez y López, 2020; Gutiérrez-Saldivia et al., 2019) que den cuenta de su implementación en el sistema educativo y su pertinencia. En este trabajo entendemos el DUA como un enfoque metodológico conformado por un conjunto de principios, pautas y puntos de verificación, que permiten diseñar un currículum accesible para maximizar las oportunidades de aprendizaje de todos los estudiantes (Meyer et al., 2014).

Con respecto a la revisión de la literatura, hay que señalar que existe una mayor producción de trabajos teóricos que realizan una reflexión crítica sobre la importancia del DUA y escasos estudios empíricos (Alba et al., 2015; Coy et al., 2014; Mangiatordi y Serenelli, 2013; Roberts et al., 2011). Otro antecedente, es el planteado por Rao et al. (2014), quienes señalan que la implementación del DUA se considera novedoso. Esto por que, existen estudios que develan que su incorporación en la educación superior favorece la accesibilidad y el aprendizaje de todos los estudiantes (Dean et al., 2016; Diez y Sánchez, 2015; Rao y Tanners, 2011; Parker et al., 2008), y particularmente en la formación del profesorado (Moore et al., 2018). En la educación superior destacamos la investigación realizada por Parker et al. (2008), quienes constataron que la implementación del DUA, en un curso e-learning, favoreció la autonomía en el aprendizaje y facilitó un ambiente de respeto en el aula virtual. Otra contribución en este ámbito es la investigación desarrollada por Rao y Tanners (2011), en un curso de formación e-learning dirigido a profesores, quienes concluyen que los recursos multimodales y actividades breves basadas en el DUA resultaron útiles para el aprendizaje de los contenidos. Asimismo, cabe señalar que los resultados del estudio de Yuval et al. (2004) demuestra que la implementación del diseño universal en la educación superior mejoró significativamente las actitudes de los estudiantes hacia el aprendizaje, su rendimiento académico y, además, benefició un entorno de aprendizaje para estudiantes con discapacidades de aprendizaje.

La implementación del DUA se realiza en el curso herramientas de acceso para el aprendizaje del lenguaje y las matemáticas I. En este curso en el ámbito de las matemáticas los estudiantes deben lograr tres resultados de aprendizaje: 1) evaluar psicopedagógicamente en la asignatura de matemática de 1 a 4 básico; 2) diseñar situaciones de enseñanza en el área de matemáticas pertinentes al nivel curricular de estudiantes con necesidades educativas especiales (NEE) que cursan de primero a cuarto año de educación básica; 3) Diseñar respuestas educativas pertinentes, considerando estrategias de apoyo que permitan potenciar las habilidades de estudiantes con NEE. Finalmente, con el propósito de responder a los resultados de aprendizaje, mejorar las prácticas docentes de las profesoras del curso -específicamente del área de las matemáticas- y fortalecer la formación de futuros profesores de educación especial, se incorporó la metodología DUA.

\section{OTROS ANTECEDENTES}

El diseño universal surge en Estados Unidos (EEUU), en la década del 70, desde el campo de la arquitectura con Ron Mace de la Universidad de Carolina del Norte. Su propósito fue promover el diseño de entornos y 
productos que pudiesen ser utilizados por todos, sin necesidad de una adaptación para las personas en situación de discapacidad (Alba et al., 2015). En el campo educativo, se transfieren los postulados y se propone el DUA cuando se reautoriza en 1997 de la Ley de Ayuda para Personas con Discapacidad Educativa (Individuals with Disabilities Education Act IDEA) de EEUU (Edyburn, 2010). Así, este enfoque se comienza a emplear en la educación, siendo el Center for Applied Special Technology CAST en Massachusetts quienes desarrollaron investigaciones en el área. Este centro constató que las barreras en el aprendizaje y la participación no son inherentes a los estudiantes, sino que emergen por la interacción de éstos con los métodos, estrategias y recursos de enseñanza (Meyer et al., 2014).

Es en este contexto, el CAST propone el DUA como enfoque para la atención de la diversidad en la educación. Esto implica que las instituciones educativas desarrollen planes de estudios y materiales accesibles, es decir que todos los estudiantes tengan acceso independiente de sus habilidades y necesidades, y así facilitar la inclusión especialmente de aquellos estudiantes que si bien no presentan necesidades educativas especiales no tienen éxito en la escuela (Katz, 2015). Cabe destacar que, desde las investigaciones realizadas por el CAST, el DUA se ha constituido en un marco científicamente válido que tiene como principal objetivo mejorar los procesos de aprendizaje mediante tecnologías y recursos didácticos que permitan flexibilidad y accesibilidad al currículum por parte de todos los estudiantes (Alba et al., 2015). Actualmente, en Chile como en otros países, este enfoque es un referente teórico que ha inspirado la creación de políticas educativas para atender a la diversidad. Esto debido a que se ha demostrado que su uso en las aulas facilita el acceso, la participación y el aprendizaje de todos los estudiantes (King-Sears, 2009; Kortering et al., 2008).

La estructura del DUA se basa en los avances y conocimientos de la neurociencia cognitiva sobre el aprendizaje. En este sentido, los investigadores señalan que en el aprendizaje actúan tres redes neuronales (Rose y Strangman, 2007). Las redes de reconocimiento, encargadas de percibir la información, facilitan al estudiante identificar y comprender ideas y conceptos; las redes estratégicas, especializadas en generar y supervisar los patrones motores y mentales, facilitan la planificación y ejecución de las acciones y habilidades; y las redes afectivas, encargadas de evaluar los patrones y asignarles un significado emocional, generan la motivación y compromiso en el proceso de aprendizaje (Rose et al., 2006).

A partir de estas tres redes neuronales, Meyer et al. (2014) proponen tres principios básicos, que incluyen 9 pautas de trabajo y 31 puntos de verificación para ser incorporados en el diseño de la enseñanza. El primer principio se refiere a proporcionar múltiples formas de implicación para permitir que los estudiantes encuentren su incentivo y se motiven en el proceso de aprendizaje (el por qué del aprendizaje). El segundo principio implica proporcionar múltiples formas de representación de la información, lo que aporta mayores opciones de acceso al aprendizaje, considerando que los estudiantes son distintos en la forma en que perciben y comprenden la información (el qué del aprendizaje). Finalmente, el tercer principio se refiere a proporcionar múltiples formas de acción y expresión, que permitan a todos los estudiantes interactuar con la información y demostrar lo aprendido según sus preferencias y capacidades (el cómo del aprendizaje). En relación con las pautas del DUA, el CAST las define como estrategias educativas que tienen una organización jerárquica, desde procesos cognitivos más sencillos y de fácil implementación hasta los más complejos (Meyer et al., 2014). En tanto, los puntos de verificación detallan con mayor precisión las estrategias contenidas en las pautas de cada principio del DUA.

Finalmente, se debe destacar que el DUA es considerado como una buena práctica inclusiva, ya que se orienta a abordar y eliminar aquellas barreras de acceso presentes en currículos que son inflexibles, lo que no implica la realización de ajustes posteriores a la implementación de las situaciones de aprendizaje, siendo una metodología que aporta tanto al aprendizaje de los estudiantes como a su participación (Sánchez-Gómez y López, 2020).

\section{METODOLOGÍA}

El diseño es investigación-acción ya que el propósito fue mejorar la práctica docente (Labra et al., 2005), a partir del rediseño del curso herramientas de acceso para el aprendizaje del lenguaje y las matemáticas I, incorporando estrategias del DUA. Los objetivos del estudio son: 1) identificar las estrategias del DUA más valoradas por los estudiantes en el curso herramientas de acceso para el aprendizaje del lenguaje y las matemáticas I; y 2) describir los facilitadores y obstaculizadores para el aprendizaje en la implementación de las estrategias del DUA.

\section{Fase de rediseño del curso}

El rediseño del curso herramientas de acceso para el aprendizaje del lenguaje y las matemáticas I se realizó a partir de la experiencia de las profesoras -quienes habían realizado previamente el curso-, y el análisis de la guía de aprendizaje del área de matemáticas del curso del año 2016. La guía de aprendizaje es un recurso 
pedagógico que proporciona los lineamientos necesarios que el estudiante debe conocer para lograr los resultados de aprendizaje definidos en el curso; entre los elementos que incluye destacamos las competencias, resultados de aprendizaje, contenidos, actividades evaluativas, metodologías de enseñanza, cronograma de actividades y referencias bibliográficas del curso.

A partir del análisis realizado se identificó lo siguiente: a) predominancia del uso de una metodología de enseñanza tradicional, correspondientes a clases magistrales, utilizadas en versiones anteriores del curso, la que no siempre facilitó el acceso a los saberes por parte de todos los estudiantes; b) las formas de representación de los contenidos utilizadas eran decisiones propias de los profesores y no respondían a principios de accesibilidad; c) el cronograma de trabajo era general, se entregaba al inicio del semestre, y contemplaba las semanas de clases y las actividades genéricas para las horas de clases presenciales, mixtas y autónomas; d) en general los estudiantes que realizaron en años anteriores el curso presentaban percepciones de las matemáticas negativas y un sentido de competencia bajo.

En relación con el análisis realizado por las profesoras y las premisas del DUA planteadas por Meyer et al. (2014) respecto a ofrecer múltiples formas de implicancia, múltiples formas de representación de la información, y múltiples formas de expresión y acción, es que se han seleccionado a partir de la estructura del DUA que se muestra en la Figura 1 las estrategias específicas -es decir pautas y puntos de verificaciónpara su implementación en el curso.

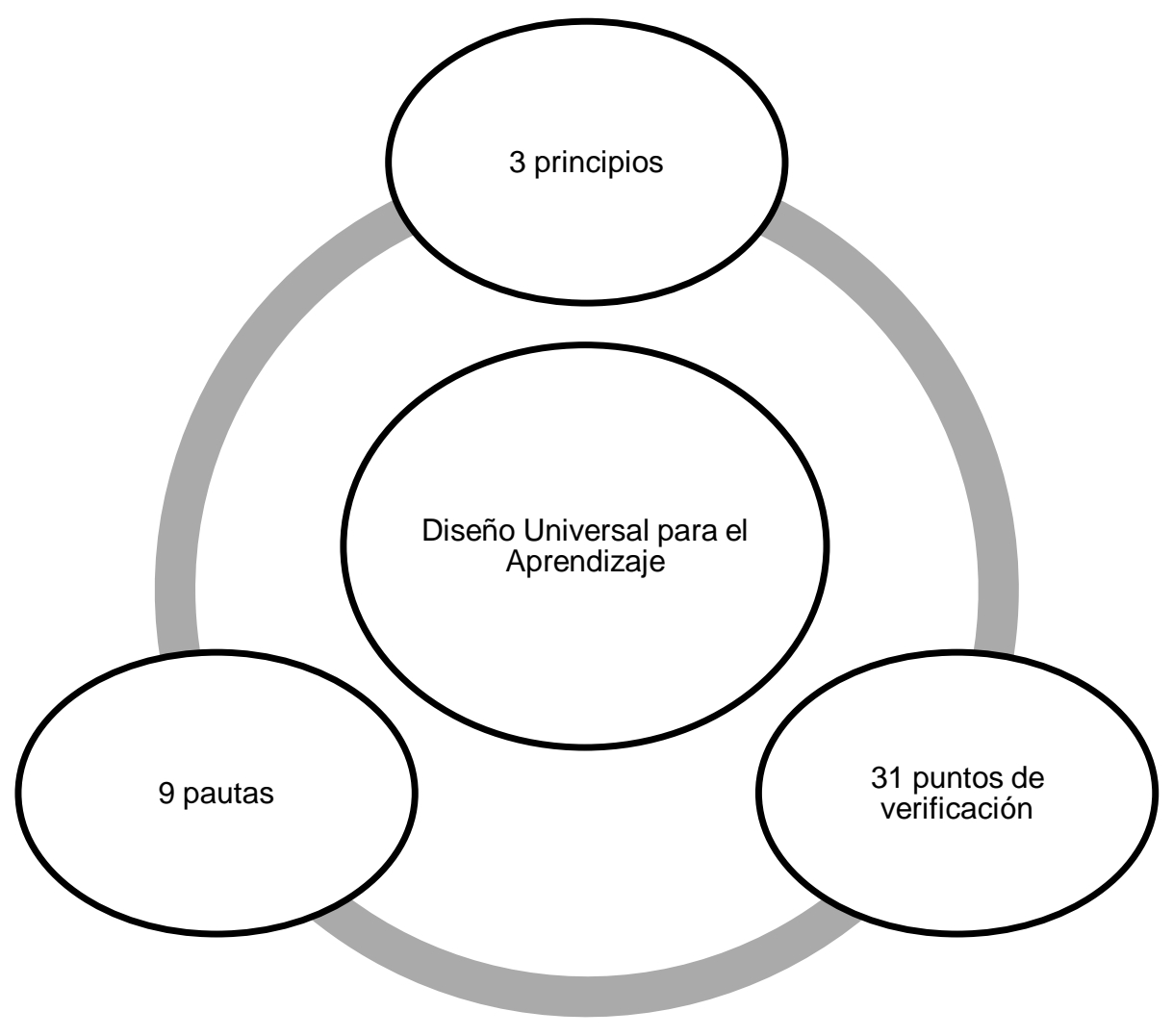

Fig. 1: Estructura del Diseño Universal para el Aprendizaje. (Adaptado de Meyer et al., 2014).

En concordancia con lo anterior, en la Tabla 1 se muestran las estrategias del DUA seleccionadas en el curso, además de los recursos pedagógicos que permitieron su puesta en práctica en las clases, y que para efectos de la recogida de información se denominan como "estrategias para la implementación del DUA". La Tabla 1 muestra que para proporcionar múltiples formas de implicación en el curso se han seleccionado dos pautas y dos puntos de verificación; además para ofrecer múltiples formas de representación se han seleccionado tres pautas y cinco puntos de verificación; y para ofrecer múltiples formas de acción y expresión se han seleccionado dos pautas y cinco puntos de verificación. En total, se han seleccionado 7 pautas y 12 puntos de verificación, que fueron implementados a partir del uso de 7 estrategias durante un semestre académico. Luego, se presenta una descripción de los siete recursos pedagógicos que permitieron su puesta en práctica en el curso. 
Tabla 1: Matriz especificaciones de estrategias del diseño universal para el aprendizaje seleccionadas para el curso (Adaptado de Meyer et al., 2014).

\begin{tabular}{|c|c|c|}
\hline Principio DUA & Estrategias del DUA: Pauta y punto de verificación DUA & $\begin{array}{l}\text { Estrategias para la } \\
\text { implementación del DUA }\end{array}$ \\
\hline $\begin{array}{l}\text { Proporcionar múltiples } \\
\text { formas de implicación }\end{array}$ & $\begin{array}{l}\text { Pauta: Proporcionar opciones para mantener el esfuerzo y la } \\
\text { persistencia } \\
\text { Punto de verificación: Resaltar la relevancia de las metas y } \\
\text { los objetivos } \\
\text { Pauta: Proporcionar opciones para captar el interés } \\
\text { Punto de verificación: Minimizar la sensación de inseguridad } \\
\text { y las distracciones }\end{array}$ & \multirow[t]{3}{*}{$\begin{array}{l}\text { Ruta de trabajo clase a } \\
\text { clase }\end{array}$} \\
\hline $\begin{array}{l}\text { Proporcionar múltiples } \\
\text { formas de } \\
\text { representación }\end{array}$ & $\begin{array}{l}\text { Pauta: Proporcionar opciones para la comprensión } \\
\text { Punto de verificación: Guiar el procesamiento de la } \\
\text { información, la visualización y la manipulación }\end{array}$ & \\
\hline $\begin{array}{l}\text { Proporcionar múltiples } \\
\text { formas de acción y } \\
\text { expresión }\end{array}$ & $\begin{array}{l}\text { Pauta: Proporcionar opciones para las funciones ejecutivas. } \\
\text { Punto de verificación: Apoyar la planificación y el desarrollo } \\
\text { de estrategias } \\
\text { Punto de verificación: Aumentar la capacidad para hacer un } \\
\text { seguimiento de los avances }\end{array}$ & \\
\hline $\begin{array}{l}\text { Proporcionar múltiples } \\
\text { formas de } \\
\text { representación }\end{array}$ & $\begin{array}{l}\text { Pauta: Proporcionar opciones para la comprensión } \\
\text { Punto de verificación: Activar o sustituir los conocimientos } \\
\text { previos } \\
\text { Pauta: Proporcionar opciones para la percepción } \\
\text { Punto de verificación: Ofrecer opciones que permitan la } \\
\text { modificación y personalización en la presentación de la } \\
\text { información. }\end{array}$ & Material preparatorio \\
\hline $\begin{array}{l}\text { Proporcionar múltiples } \\
\text { formas de } \\
\text { representación }\end{array}$ & $\begin{array}{l}\text { Pauta: Proporcionar opciones para el lenguaje, las } \\
\text { expresiones matemáticas y los símbolos } \\
\text { Punto de verificación: Ilustrar la información a través de } \\
\text { múltiples medios } \\
\text { Pauta: Proporcionar opciones para la percepción } \\
\text { Punto de verificación: Ofrecer alternativas para la } \\
\text { información auditiva } \\
\text { Punto de verificación: Ofrecer alternativas para la } \\
\text { información visual }\end{array}$ & \multirow[t]{2}{*}{$\begin{array}{l}\text { Material didáctico } \\
\text { manipulable y material } \\
\text { audiovisual }\end{array}$} \\
\hline $\begin{array}{l}\text { Proporcionar múltiples } \\
\text { formas de acción y } \\
\text { expresión }\end{array}$ & $\begin{array}{l}\text { Pauta: Proporcionar opciones para la expresión y la } \\
\text { comunicación } \\
\text { Punto de verificación: Usar múltiples herramientas para la } \\
\text { construcción y la composición }\end{array}$ & \\
\hline $\begin{array}{l}\text { Proporcionar múltiples } \\
\text { formas de } \\
\text { representación }\end{array}$ & $\begin{array}{l}\text { Pauta: Proporcionar opciones para la percepción } \\
\text { Punto de verificación: Ofrecer alternativas para la } \\
\text { información auditiva } \\
\text { Punto de verificación: Ofrecer alternativas para la } \\
\text { información visual }\end{array}$ & Clases grabadas \\
\hline $\begin{array}{l}\text { Proporcionar múltiples } \\
\text { formas de acción y } \\
\text { expresión }\end{array}$ & $\begin{array}{l}\text { Pauta: Proporcionar opciones para la expresión y } \\
\text { comunicación } \\
\text { Punto de verificación: Usar múltiples medios de } \\
\text { comunicación }\end{array}$ & $\begin{array}{l}\text { Foro virtual } \\
\text { Wiki }\end{array}$ \\
\hline $\begin{array}{l}\text { Proporcionar múltiples } \\
\text { formas de acción y } \\
\text { expresión }\end{array}$ & $\begin{array}{l}\text { Pauta: Proporcionar opciones para la expresión y } \\
\text { comunicación } \\
\text { Punto de verificación: Usar múltiples medios de } \\
\text { comunicación } \\
\text { Pauta: Proporcionar opciones para las funciones ejecutivas } \\
\text { Punto de verificación: Facilitar la gestión de información y de } \\
\text { recursos }\end{array}$ & Toma de apuntes \\
\hline
\end{tabular}




\section{Ruta de trabajo clase a clase:}

Es una planificación semanal de las actividades del curso, la que era enviada a los estudiantes una vez a la semana a través de la plataforma virtual Moodle e-learning Educa, con 48 horas de antelación a la clase presencial. Esta ruta clase a clase incorpora el objetivo de la clase, las actividades que se realizarían, y que fueron organizadas en tres momentos: antes de la clase, durante la clase y después de la clase. En las actividades antes de la clase se informaban los materiales que podían ser utilizados por los estudiantes y que se denominan material preparatorio; además se indican las actividades que se realizarían en la clase presencial, y las actividades a realizar después de esta. Cabe destacar que a través de este recurso pedagógico se presentan los objetivos de cada clase, además se busca minimizar las inseguridades y las distracciones, informando a los estudiantes de la totalidad de las actividades de la semana. Además, permite apoyar la planificación de estrategias de aprendizaje y aumentar en los estudiantes su capacidad para hacer un seguimiento de sus avances.

\section{Material preparatorio}

Son recursos asociados a presentaciones de PowerPoint y lecturas preparatorias respecto de los temas que se trabajan en la clase presencial. Los estudiantes cada semana tenían acceso a los materiales desde la plataforma virtual Moodle e-learning Educa, previo a las clases presenciales, con un mínimo de 48 horas antes. Acceder previamente a los temas que se abordarían en las clases tenía como propósito que los estudiantes tuviesen un conocimiento previo antes de la clase presencial para potenciar la comprensión de los contenidos que se trabajaban en casa sesión. Además, estos recursos eran compartidos en formato ppt y doc respectivamente, lo que permite la personalización de la información, ya que cada estudiante tenía la posibilidad de modificarlo.

\section{Material didáctico manipulable}

Son materiales didácticos estructurados y no estructurados de tipo concreto que se utilizan en la enseñanza de la matemática, por ejemplo: Bloque Base 10, Tablero posicional, Caja Mackinder, Tabla de Séguin, Tubos conectables, Contadores, Listones rojos y azules, Balanza numérica, entre otros. En el contexto del estudio estos materiales fueron usados como recursos alternativos a las explicaciones orales de las profesoras y a la información visual presentes en los textos y presentaciones PowerPoint. Se utilizaron al momento de la enseñanza de contenidos matemáticos conceptuales y procedimentales. Su incorporación tuvo como finalidad facilitar la comprensión para hacer más accesible la información a todos los estudiantes. Además, el trabajo con material didáctico permite a los estudiantes usar otras herramientas para expresar lo que están aprendido, ya que a través de este recurso pueden presentar soluciones y reconocer los procesos que se siguen para llegar a un resultado.

\section{Material audiovisual:}

Son videos que muestran segmentos de clases de matemática de educación primaria y que cuentan con subtítulos en castellano y audio. Al igual que el material didáctico manipulable los videos de clases se utilizaron como recursos alternativos para facilitar la comprensión y hacer más accesible la información a todos los estudiantes, utilizándose otras formas alternativas de presentación, pues incluye alternativas para la información visual y auditiva. En este caso particular, lo alternativo a lo auditivo son los subtítulos en castellano, y lo alternativo a lo visual es el audio. Cabe destacar, que usar segmentos de clases reales permite anticipar a los estudiantes la realidad de enseñar matemáticas e identificar la complejidad de la tarea. A su vez, este material permitió analizar y reflexionar sobre la enseñanza de las matemáticas, el rol del profesor, las acciones que emplea para enseñar y el rol de los estudiantes.

\section{Clases grabadas}

Todas las clases presenciales del curso fueron grabadas en video y compartidas con los estudiantes mediante la herramienta Google Drive. Contar con las grabaciones de las clases presenciales tenía como objetivo ofrecer otras formas de representar los contenidos abordados en las clases, proporcionando alternativas para la percepción posterior a la clase, ya que estos vídeos contaban con audio, es decir información alternativa a lo visual, y subtítulos en castellano, correspondiente a información alternativa a lo auditivo. Además, esta estrategia se orientó a facilitar el aprendizaje autónomo, ya que permite a los estudiantes acceder nuevamente a la clase para estudiar y profundizar en los contenidos.

\section{Foro virtual y Wiki}

Son recursos de la plataforma Moodle e-learning Educa. El foro permite a los estudiantes discutir sobre un tema o problemática asociada al área de matemáticas. Dicha actividad es planificada previamente por el equipo de profesoras, donde se plantean preguntas orientadoras y se proporcionan lecturas complementarias. 
Con respecto a la Wiki, este recurso les permite a los estudiantes añadir y editar una página web de forma colaborativa sobre un tema o problemática asociada al curso. El uso del foro y wiki tiene como objetivo proporcionar a los estudiantes diferentes formas de comunicar lo aprendido, ya que pueden incluir texto, audios, vídeos e imágenes, aumentando las formas de expresión de lo aprendido.

\section{Toma de apuntes}

Esta estrategia consiste en que un grupo de estudiantes tomen apuntes en las clases presenciales y, luego, los compartan a través de la plataforma Moodle e-learning Educa con sus compañeros. Se promovía el uso de diversas formas de expresión de lo aprendido y facilitar la gestión de información, donde los estudiantes eran quienes seleccionaban la forma de registrar las ideas principales abordadas en clases. El foco estaba centrado en que cada estudiante utilizara la forma de expresión que más le acomodara, por ejemplo, a través de mapas mentales, pictogramas, dibujos, texto escrito, u otras formas de comunicar la información.

\section{Muestra}

La muestra del estudio se compone de 80 estudiantes de la carrera de pedagogía en educación diferencial de una universidad ubicada al sur de Chile, que cursan la asignatura "herramientas de acceso para el aprendizaje del lenguaje y las matemáticas I". La selección de la muestra se realizó mediante la técnica de muestreo no probabilístico de tipo intencional, donde todos los estudiantes inscritos en el curso son fuente importante de información de acuerdo con los objetivos del estudio. Con respecto a las características de los participantes hay que señalar que ingresaron a la universidad el año 2015, y en el momento en que se realizó este estudio se encontraban cursando el quinto semestre de la carrera, correspondiente al tercer año de su itinerario formativo. Además, hay que señalar que de los 80 participantes $97 \%$ eran mujeres y $3 \%$ hombres. Además, de la muestra el $61 \%$ los estudiantes egresaron de establecimientos de educación secundaria particulares subvencionados, el $37 \%$ de establecimientos municipales y el $2 \%$ de establecimientos privados. Cabe destacar que en Chile existen tres tipos de establecimientos educativos -particulares subvencionados, municipales y privados-, siendo los establecimientos municipales y particulares subvencionados los que reciben financiamiento estatal, bajo la modalidad de una subvención -correspondiente a un aporte económico- mensual por alumno matriculado.

\section{Instrumentos}

Para la recogida de información, se diseñaron dos instrumentos ad hoc, una escala de valoración y un cuestionario con preguntas abiertas, esto debido a la naturaleza del estudio y los objetivos. Para el objetivo 1 -correspondiente a identificar las estrategias DUA más valoradas por los estudiantes- se diseñó una escala de valoración. Esta escala esta conformada por dos apartados y 10 ítems. El primer apartado considera el título del instrumento, finalidad de este, datos de identificación personal de los participantes asociado a fecha, género, edad y tipo de establecimiento educativo de educación secundaria del que egresó, e instrucciones para contestar la escala. El segundo apartado considera 10 ítems, donde los participantes tuvieron que valorar las estrategias para la implementación del DUA según su relevancia y aporte en su proceso de aprendizaje. El formato de respuesta que se utilizó es de tipo Likert con una escala de 10 puntos, siendo 1 el valor más negativo y 10 el más positivo. En primer lugar, los estudiantes debieron valorar la relevancia, donde tuvieron que ordenar las estrategias del 1 al 10, siendo 1 la menos importante y 10 la más importante. En segundo lugar, debieron valorar el aporte de las estrategias en su proceso de aprendizaje, siendo 1 la que menos aportó y 10 la que más aportó. Para el objetivo 2 -describir los facilitadores y obstaculizadores en la implementación del DUA- se utilizó un cuestionario de preguntas abiertas, donde los participantes respondieron a dos preguntas a partir de su experiencia en el curso. Estas preguntas estaban orientadas a conocer sobre los aspectos que han favorecido el aprendizaje de los estudiantes en el curso, los aspectos que han sido obstaculizadores, y las estrategias que favorecieron u obstaculizaron su aprendizaje.

\section{Validación de los instrumentos:}

La escala de valoración y cuestionario de preguntas abiertas fueron construidos en relación con los objetivos del estudio y la revisión de la literatura realizada. Además, ambos instrumentos fueron sometidos a validación de jueces expertos y prueba piloto. La validación de jueces expertos fue realizada por cuatro doctores especialistas en el área de metodologías de investigación en educación, quienes evaluaron ambos instrumentos según criterios de relevancia, claridad y pertinencia de los ítems y preguntas. Posterior a esto se realizaron correcciones a ambos instrumentos según sugerencias de los expertos, lo que permitió contar con una nueva versión de los instrumentos, quedando la escala de valoración con un total de 10 ítems, y el cuestionario abierto con dos preguntas. Luego del juicio de expertos se aplicaron ambos instrumentos a una pequeña muestra para probar su claridad, pertinencia y condiciones de aplicación. Finalmente, hay que señalar que para la escala de valoración se cálculo adicionalmente el Alfa de Cronbach, obteniéndose un alfa de 0,8, considerado adecuado (Solis-Esparllargas y Barreto-Tovar, 2020). 


\section{Recogida de información y análisis de datos}

Antes de implementar el DUA en el curso se informó sobre la investigación a la dirección de carrera y a los estudiantes del curso. Posteriormente, se implementó la metodología del DUA entre los meses de marzo y junio del año 2017. Luego, en el mes de julio del mismo año se recogieron los datos. Con respecto a la escala de valoración, hay que señalar que fue aplicada en una sesión presencial que duró aproximadamente 30 minutos; mientras que el cuestionario de preguntas abiertas fue aplicado en una modalidad virtual a través de un foro en la plataforma Moodle e-learning Educa. Cabe destacar, que la participación de los estudiantes fue voluntaria, y que antes de la aplicación de los dos instrumentos, se solicitó el consentimiento informado a los participantes, donde se garantizó el anonimato y la confidencialidad de la información.

El análisis de los datos se realizó desde una perspectiva cuantitativa y cualitativa, según la naturaleza de los datos. Desde una perspectiva cuantitativa y en concordancia con el objetivo 1 del estudio, se obtuvieron a través del programa Microsoft Excel las puntuaciones medias otorgadas por los estudiantes en la escala de valoración a las estrategias implementadas en el curso, esto en relación con la relevancia que le dan a las estrategias y el aporte de estas en su aprendizaje. Desde la perspectiva cualitativa y en concordancia con el objetivo 2 del estudio se realizó análisis de contenido, con el propósito de identificar temas centrales y subtemas, que se muestran en la Tabla 2. El análisis de contenido contempló en un primer momento el levantamiento de códigos mediante la lectura preliminar de las respuestas que dieron los estudiantes en el cuestionario de preguntas abiertas, obteniéndose 30 códigos. Luego, se redujeron estos códigos mediante el agrupamiento según su relación y dependencia, estableciendo dos categorías centrales. La categoría denominada facilitadores para el aprendizaje agrupa las subcategorías materiales para el aprendizaje, características de las profesoras y estrategias de enseñanza diversas, y expone a partir de la experiencia de los estudiantes en el curso rediseñado con DUA aquellos aspectos que consideran que aportaron a su aprendizaje. La categoría obstaculizadores para el aprendizaje, agrupa las subcategorías organización y aspectos técnicos, las que dan cuenta de aquellos aspectos que los estudiantes consideran que afectaron negativamente en su aprendizaje. Cabe destacar que los resultados del análisis de contenido se organizaron en la Figura 2 y 3 , que se presentan en el apartado de resultados.

Tabla 2: Categorías, subcategorías y componentes de las subcategorías del análisis de contenido.

\begin{tabular}{|l|l|l|}
\hline Categorías & Subcategorías & Componentes \\
\hline Facilitadores para el aprendizaje & Material para el aprendizaje & $\begin{array}{l}\text { Concreto } \\
\text { Audiovisual } \\
\text { Preparatorio }\end{array}$ \\
\cline { 2 - 3 } & Características de las profesoras & $\begin{array}{l}\text { Personales } \\
\text { Profesionales }\end{array}$ \\
\cline { 2 - 3 } & Estrategias de enseñanza diversas & $\begin{array}{l}\text { Estrategias presenciales } \\
\text { Estrategias no presenciales }\end{array}$ \\
\hline Obstaculizadores para el aprendizaje & Organización & $\begin{array}{l}\text { Extensión de clases } \\
\text { Horario de clases }\end{array}$ \\
\cline { 2 - 3 } & Aspectos técnicos & $\begin{array}{l}\text { Calidad clases } \\
\text { Tamaño presentaciones }\end{array}$ \\
\hline
\end{tabular}

\section{RESULTADOS}

En los resultados que se presentan a continuación, se incluyen extractos de las respuestas de los estudiantes recogidos a través del cuestionario de preguntas abiertas. Cabe destacar que los extractos de respuestas que se incluyen son nombrados como E1, E2, E3, ..., E80. En relación con el objetivo 1 "identificar las estrategias DUA más valoradas por los estudiantes en el curso herramientas de acceso para el aprendizaje del lenguaje y las matemáticas I", en la Tabla 3, se muestra que entre las estrategias más valoradas por los estudiantes destacan aquellas que se utilizaron antes de las clases presenciales: el Material preparatorio $(9,5)$, que considera las presentaciones de PowerPoint con el contenido detallado de la sesión de trabajo; las lecturas previas $(7,9)$, que amplían la información contenida en la presentación, y la ruta de trabajo $(7,0)$, que entrega información relacionada con el objetivo de aprendizaje y organización de las actividades.

Además, fueron altamente valoradas aquellas estrategias utilizadas en las clases presenciales, tales como el Material didáctico manipulable $(9,5)$, que se usó para ilustrar los contenidos a través de múltiples medios y para modelar el uso de las estrategias y el aprendizaje del contenido, como también el material audiovisual $(8,2)$, y la toma de apuntes $(6,8)$. En menor medida, se valoran las estrategias que se utilizan después de las sesiones presenciales, como las Clases grabadas $(5,9)$, que se sube a un espacio de almacenamiento de archivos compartido, y las estrategias virtuales asociadas a Wiki $(5,4)$, y Foro virtual $(5,1)$ que permiten realizar talleres individuales y grupales, mediante la plataforma Moodle e-learning Educa. 
Además, se observa una relación entre las estrategias que más aportan al aprendizaje y la relevancia. Por ejemplo, en material didáctico se obtiene una puntuación media de 9,5 de aporte al aprendizaje y 8,8 de relevancia. Del mismo modo el material preparatorio presentaciones de PowerPoint obtiene una puntuación media de aporte al aprendizaje de 9,5 y 8,5 de relevancia. En menor medida se valoran las clases grabadas con una puntuación media de aporte de 5,9 y 4,2 de relevancia, además el foro virtual que obtiene una puntuación media de 5,1 en aporte y 3,5 en relevancia, y la wiki que obtuvo una puntuación media de 5,4 en aporte y de 2,9 en relevancia.

Tabla 3: Valoración media del aporte y relevancia de las estrategias DUA.

\begin{tabular}{|l|c|c|}
\hline Estrategias para la implementación de DUA & Aporte & Relevancia \\
\hline Material didáctico manipulable & 9,5 & 8,8 \\
\hline Material preparatorio: presentaciones de PowerPoint & 9,5 & 8,5 \\
\hline Material audiovisual & 8,2 & 7,0 \\
\hline Material preparatorio: lecturas & 7,9 & 6,5 \\
\hline Ruta de trabajo clase a clase & 7,0 & 7,1 \\
\hline Toma de apuntes & 6,8 & 5,2 \\
\hline Clases grabadas & 5,9 & 4,2 \\
\hline Wiki & 5,4 & 2,9 \\
\hline Foro virtual & 5,1 & 3,5 \\
\hline
\end{tabular}

Los resultados dan cuenta que los estudiantes valoran para su aprendizaje estrategias vinculadas a proporcionar múltiples formas de representación de la información, tales como el uso de Material preparatorio, Material didáctico manipulable, Material audiovisual de clases reales de matemática y Ruta de trabajo clase a clase. Por ejemplo, el estudiante E22 señaló que la estrategia que más le favoreció "(...) fue el material audiovisual y el trabajo con el material concreto, ya que los videos entregaban información más visual de lo tratado, por ende, activaba nuestras funciones cognitivas; en cuanto al material concreto, este me ayudó a comprender cómo se puede trabajar con los estudiantes considerando, además, los pasos y las instrucciones que se deben dar para que toda actividad realizada pueda ser acertada". Adicionalmente, los alumnos valoran, para su aprendizaje, estrategias vinculadas a proporcionar múltiples formas de acción y expresión, como, por ejemplo, el uso de Material didáctico manipulable, que permite expresar el aprendizaje a través de la acción. Esto se constata en la siguiente respuesta del estudiante E12: "Por otro lado, la presentación y el trabajo con material concreto permitieron clarificar los contenidos que se iban abordando, como también teniendo la posibilidad de manipularlos realizando ejercicios o bien simulando nuestra enseñanza en pequeñas situaciones de aprendizajes".

En cuanto al objetivo 2 "describir los facilitadores y obstaculizadores para el aprendizaje en la implementación de las estrategias del DUA", se informa que emergen dos categorías y cinco subcategorías, que se presentan en dos redes conceptuales denominadas Figura 2 y Figura 3. En la Figura 2 se muestra la categoría Facilitadores para el aprendizaje y las subcategorías Material para el aprendizaje, Características de las profesoras y Estrategia de enseñanza diversas.

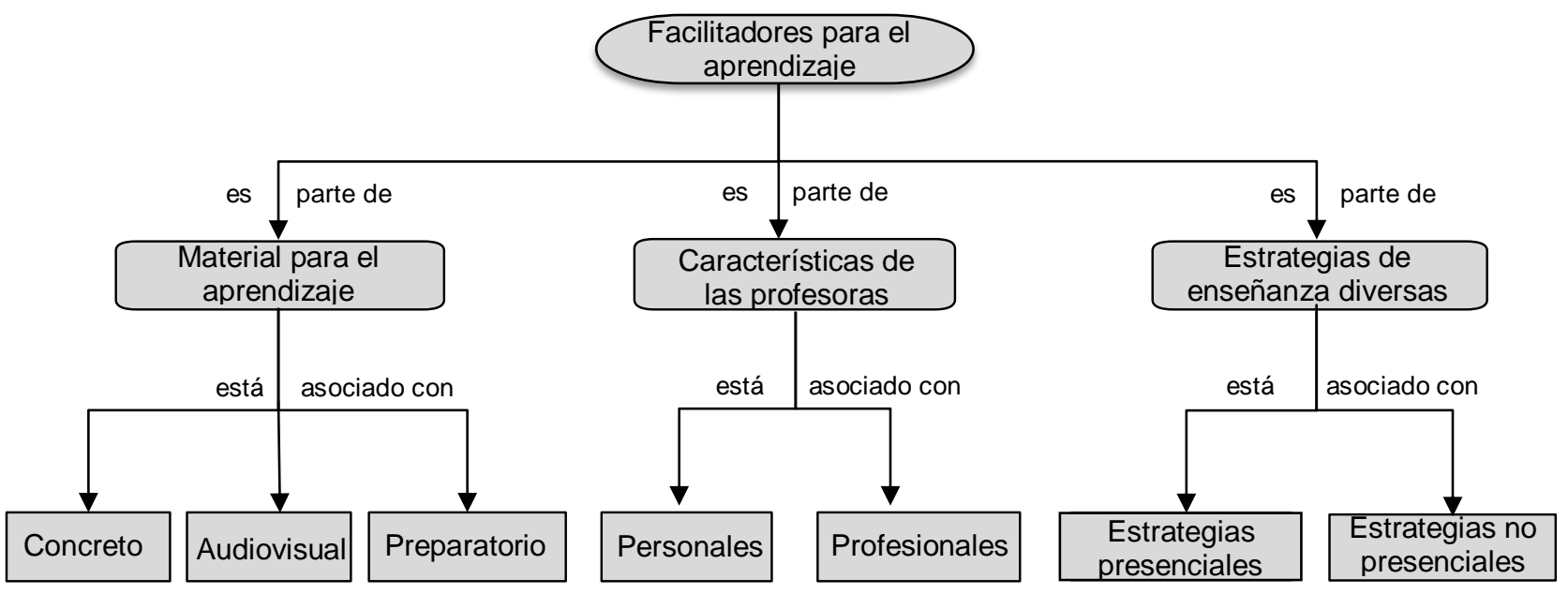

Fig. 2: Facilitadores en la implementación del curso rediseñado con DUA. 
En relación con la subcategoría Material para el aprendizaje, los estudiantes consideran que la utilización de diversos materiales didácticos, preferentemente concretos y audiovisuales favorece tanto la comprensión del contenido conceptual como su aplicación en la enseñanza a nivel procedimental. Esto se manifiesta en la respuesta del estudiante E34: “(...) el uso de material concreto me permitió aterrizar el contenido a la vida real”. Asimismo, manifiestan que utilizar materiales didácticos en las clases presenciales les permitió reconocer sus componentes y los procedimientos para una adecuada enseñanza de la matemática. Por ejemplo, la estudiante E7 manifestó "(...) lo que más favoreció mi aprendizaje fue la utilización de material concreto, porque tuve la posibilidad de usar el material, ver sus componentes y planificar una actividad, ya conociendo como se utiliza (...)".

Cabe destacar que compartir Material preparatorio como la ruta de trabajo, presentaciones de PowerPoint y lecturas complementarias, de forma previa a la clase, permitió a los estudiantes acceder a la información y organizar el contenido anticipadamente. Esto se observa en la siguiente respuesta: "(...) el material preparatorio como PPT, me permitió poder organizar mi aprendizaje previamente y poder conocer los contenidos que se iban a abordar en la clase, teniendo la posibilidad de anticipar mis dudas que luego podían ser respondidas en conjunto con la profesora (...)" (E15). Como lo expresa la respuesta del estudiante, se valora el material preparatorio, ya que permite identificar dudas respecto del contenido, elaborar preguntas e investigar sobre la temática.

La subcategoría denominada Características de las profesoras esta conformada por los componentes Personales y Profesionales. Con respecto a las características personales de las docentes, los estudiantes manifiestan que estas presentan una actitud cercana, amable y carismática, que favorece un clima ameno y que promueve el aprendizaje y la participación. A su vez, se valora que las profesoras estén constantemente motivadas por el aprendizaje de sus estudiantes, lo que convierte esta actitud en un facilitador. Esto se ilustra en la respuesta del estudiante E24 cuando señala que: “(...) facilitó mi aprendizaje la cercanía de la profesora y su constante preocupación porque mi aprendizaje sea efectivo (...)". Desde el punto de vista profesional, se destaca la disposición de las profesoras para atender consultas, retroalimentar y explicar reiteradamente el contenido, lo que favoreció el aprendizaje, dado que utilizaban diversas estrategias para explicar. Así, lo manifiesta el estudiante E21: “(...) la profesora repetía los conceptos o contenido que no me quedaban claros, ejemplificando con material concreto, pictórico y simbólico (...)".

Adicionalmente, los estudiantes destacan en la subcategoría Profesionales, la claridad de las profesoras para explicar el contenido, ya que utilizaban ejemplos cotidianos, los contextualizaban con la práctica y diversificaban estrategias para lograr los aprendizajes. Por ejemplo, la estudiante E17 manifiesta que "se aprecian explicaciones contextualizadas considerando aspectos de nuestras prácticas en diversos centros". Asimismo, la estudiante E16 explicita que "las exposiciones de clases son claras y completas, bien organizadas y existía funcionalidad al enseñar el contenido".

La última subcategoría, Estrategias de enseñanza diversas, agrupa dos componentes, denominadas: Estrategias presenciales y Estrategias no presenciales. Esta subcategoría refiere a que las profesoras utilizan diferentes estrategias en las situaciones de aprendizaje, tales como simulaciones con materiales didácticos manipulables, toma de apuntes, talleres, ejemplificaciones, manuales de aplicación y material preparatorio. Las Estrategias de enseñanza diversas se clasifican en dos tipos, presenciales, cuando el profesor trabaja en aula, y no presenciales, cuando se organizan en la plataforma virtual Moodle e-learning Educa. Con respecto a las Estrategias presenciales, se destacan como facilitadores para el aprendizaje las siguientes estrategias: (i) material didáctico manipulable, que fueron utilizados tanto por el profesor para explicar los contenidos como por los estudiantes en instancias de juego de roles y en la resolución de problemas y ejercicios matemáticos; (ii) toma de apuntes, que corresponde a los registros que los estudiantes realizan del contenido durante las clases, lo que favorece la atención dentro de la sala y permite nutrir los propios apuntes con la nueva información sistematizada por las compañeras; (iii) ejemplificaciones, que constituyen las explicaciones dialogadas y los ejemplos con material didáctico manipulable que las profesoras utilizan en la presentación del contenido para hacer aclaraciones o resolver dudas; y (iv) talleres, que son actividades grupales de aplicación del contenido. En concordancia con lo señalado, la estudiante E52 expresa lo siguiente: "(...) otro elemento que favoreció mi aprendizaje, es la utilización y ejemplificación de los contenidos con material concreto, actividades lúdicas y videos demostrativos, teniendo la posibilidad de poder trabajar con aquellos materiales en simulaciones de actividades". Asimismo, la estudiante E46 señaló "(...) otra estrategia que favoreció mi aprendizaje es que la profesora nos enseñaba a nosotros con material concreto como después les enseñaremos a nuestros propios estudiantes, lo cual se me hizo muy significativo y más sencillo de aprender, considerando que no fue solo teoría sino que también se observaban las estrategias de forma práctica".

En cuanto a las Estrategias no presenciales, se destacan las siguientes: (i) manuales de aplicación, que son instructivos que explican cómo utilizar el material didáctico manipulativo para la enseñanza de las 
matemáticas; y (ii) material preparatorio de clases, que corresponde a las presentaciones de PowerPoint y a las lecturas complementarias. De acuerdo con ello, la estudiante E64 señala que favoreció su aprendizaje "(...) la entrega de los ppt antes de la clase, ya que, al exponer la docente la materia facilitaba la comprensión, dando mayor espacio para resolver dudas (...)". Asimismo, la estudiante E74 manifestó que "a pesar de que todas las estrategias fueron muy positivas, quisiera destacar primeramente, el material preparatorio de la clase, por que me predispuso a los aprendizajes y a las actividades que se desarrollarán en estas". En suma, los resultados sobre los facilitadores para el aprendizaje que se relacionan con el uso de materiales para el aprendizaje y estrategias de enseñanza diversas dan cuenta que ofrecer múltiples formas de representación de la información es positivo para el aprendizaje, pues fue valorado como un facilitador por los estudiantes.

En la Figura 3 se muestra la categoría obstaculizadores para el aprendizaje, y las dos subcategorías Organización y Aspectos técnicos. La primera subcategoría Organización está relacionada con aspectos operativos donde se destaca en primer lugar la Extensión de clases, que se prolongaron por un periodo de 4 horas continuas, lo que dificultó la atención de las estudiantes durante toda la clase. Al respecto la estudiante E16 señala que "(...) el horario de clase, puesto que al ser 4 horas en horario de tarde perjudicaba mis procesos de atención". En segundo lugar, los Horarios de la clases, asociados al día y la hora, dificultaron la asistencia de los estudiantes y, a su vez, la comprensión de los contenidos. De este modo, la estudiante E6 plantea que "(...) el horario de clases dificulta mi asistencia y no aprendo al 100\%".

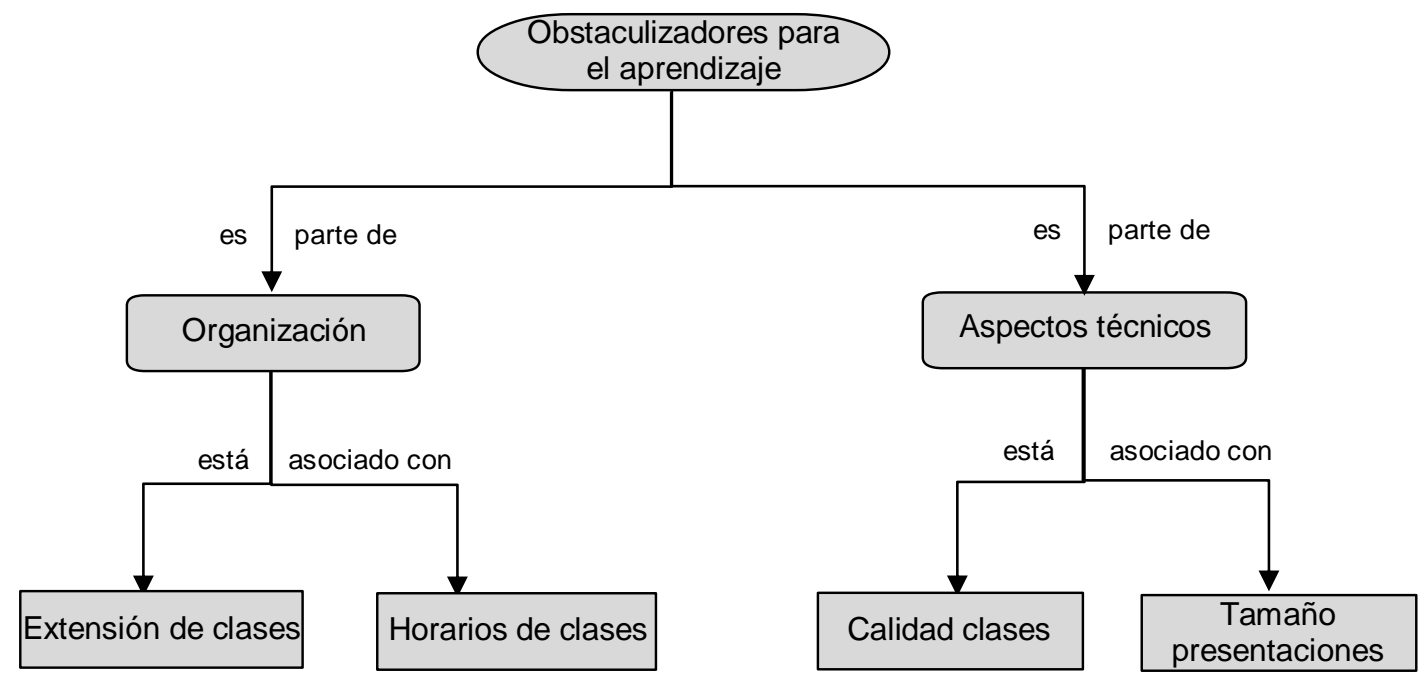

Fig. 3: Obstaculizadores en la implementación del curso rediseñado con DUA.

La segunda categoría, denominada Aspectos técnicos devela, como obstaculizadores para el aprendizaje, la Calidad de clases, entendiendo la calidad en relación con la infraestructura, como es el tamaño y acústica de la sala de clases, lo que dificultó la visión de las actividades, la participación y desenvolvimiento de los estudiantes en las clases. Esto se evidencia en la respuesta de la estudiante E7 “ (...) los obstaculizadores fueron más que nada que eran muchos alumnos, por lo que en ocasiones existían interrupciones o no se lograba oír lo que la profesora decía". Además, el Tamaño de las presentaciones de PowerPoint, que eran extensas, afectan la motivación y la concentración de los estudiantes. Del mismo modo, los videos de más de 2 GB son de difícil descarga y esto limita su uso como un recurso de aprendizaje. De acuerdo con lo anterior la estudiante E13 señala que "como obstaculizador destaco las clases expositivas, puesto que estas eran extensas y los PPT contenían mucha información (texto)". De los obstaculizadores, se destaca la extensión de las presentaciones en PowerPoint, por cuanto es un elemento del cual el profesor tiene control y se puede modificar para favorecer de mejor forma el aprendizaje de los estudiantes.

\section{DISCUSIÓN}

A continuación, se presentan los principales hallazgos del estudio según los objetivos propuestos: 1) identificar las estrategias del DUA más valoradas por los estudiantes en el curso herramientas de acceso para el aprendizaje del lenguaje y las matemáticas I; y 2) describir los facilitadores y obstaculizadores para el aprendizaje en la implementación de las estrategias del DUA.

En relación con el objetivo 1 se informa que las estrategias más valorados por los estudiantes son los materiales didácticos manipulables, materiales audiovisuales y materiales preparatorios (presentaciones PowerPoint y Lecturas). Estos recursos pedagógicos permitieron poner en práctica en el curso los puntos de verificación del DUA asociados a ilustrar a través de múltiples medios, ofrecer alternativas a la información 
visual y auditiva, y activar o sustituir los aprendizajes previos. Estas cuatro estrategias del DUA (puntos de verificación) permitieron proporcionar a los estudiantes múltiples formas de representación de la información en las clases, lo que es coincidente con los argumentos de las estudiantes, quienes plantear que el uso de diversidad de materiales y estrategias fueron un facilitador en sus aprendizajes. Este hallazgo es coherente con lo planteado por Meyer et al. (2014), quienes señalan que proporcionar múltiples formas de representación del contenido aporta un mayor y más amplio espectro de opciones para que todos los estudiantes puedan acceder al aprendizaje. Por lo tanto, el uso de variados recursos pedagógicos en el curso, y en especial el material didáctico manipulable, permitió como bien señala Alba et al. (2015) ofrecer a todos los estudiantes una "amplia variedad de formas de percibir, procesar y comprender la información" (p. 93).

Otro aspecto para destacar con respecto al objetivo 1 del estudio, es la valoración positiva por parte de los estudiantes de la ruta clase a clase, donde manifestaron que este recurso pedagógico les ayudó a planificar y organizar sus tiempos, ideas y actividades del curso. Los argumentos de los participantes del estudio son coherentes con tres de los cinco puntos de verificación del DUA que se pusieron en práctica en el aula a través del uso de la ruta clase a clase: i) apoyar la planificación y el desarrollo de estrategias, ii) aumentar la capacidad para hacer un seguimiento de los avances; y iii) guiar el procesamiento de la información, la visualización y la manipulación. De acuerdo con Rose et al. (2006) y Rose y Strangman (2007), utilizar estrategias que favorecen la activación de las redes estratégicas permite a los estudiantes establecer un plan de trabajo y definir sus propias metas y pasos para lograrlos. En este mismo orden de ideas, Alvarado et al. (2014) señalan que favorecer la planificación estratégica en los estudiantes de educación superior es esencial para que logren el éxito en el desempeño académico. Además, la ruta clase a clase aporta tanto dentro como fuera de la clase, ya que es un recurso que los estudiantes utilizan antes de la clase, donde los anticipa y prepara para aprender y participar en la clase presencial. En este sentido, Dean et al. (2016), plantea que las herramientas de enseñanza que son accesibles dentro y fuera de la clase favorecen más el aprendizaje.

En cuanto al objetivo 2, hay que señalar que se los estudiantes reconocen tres facilitadores para el aprendizaje en el curso, los que se relacionan con el uso de materiales para el aprendizaje, uso de estrategias de enseñanza diversas y las características de las profesoras. Los facilitadores que se vinculan con las subcategorías materiales para el aprendizaje y estrategias de enseñanza diversas se relacionan con las estrategias más valoradas por los estudiantes, y que se discutieron en el párrafo precedente por haber aportado al aprendizaje. Otro de los hallazgos que destacamos en los facilitadores son las características personales y profesionales de las profesoras - que si bien no reporta información directa sobre las estrategias implementadas- se releva en las voces de los estudiantes como un aspecto positivo. En esta perspectiva, hay que señalar que las características personales y profesionales de las profesoras (tales como la amabilidad, la cercanía y la disposición, domino del contenido), develan una incidencia positiva en el aprendizaje y en la participación de los estudiantes.

El estudio de Villarroel y Bruna (2017) señala que enseñar en la actualidad en la universidad requiere de ciertos conocimientos, habilidades y actitudes diferenciadas de los profesores, lo que es reconocido en este estudio por los estudiantes como facilitadores para su aprendizaje. Otro aspecto clave son las competencias transversales del profesor, que están asociadas con la interacción profesor-estudiante, ya que inciden en la efectividad y satisfacción de los estudiantes con el proceso de enseñanza-aprendizaje (Villarroel y Bruna, 2017). Este hallazgo en particular nos permite sostener que las competencias profesionales transcienden las metodologías de enseñanza. Por lo tanto, un aspecto clave que se debe considerar en la puesta en práctica del DUA son las características personales y profesionales de las profesoras, ya que diversificar las estrategias de enseñanza es relevante y aportan al aprendizaje, pero no es suficiente, existiendo otras variables en el proceso de enseñanza que inciden en el aprendizaje y que se deben considerar cuando se utiliza la metodología del DUA. En relación con los obstaculizadores asociados a la implementación de estrategias del DUA, hay que señalar que los estudiantes hacen referencia como un obstaculizador las características de dos de las estrategias-recursos pedagógicos utilizados en el curso, como es el caso de la extensión del Material preparatorio presentaciones en PowerPoint y el tamaño de los videos de las Clases grabadas, aspectos que se deben tener presente al diseñar recursos pedagógicos para la puesta en práctica del DUA.

\section{CONCLUSIONES}

Según los resultados del estudio se concluye lo siguiente:

1. La incorporación del enfoque DUA en el curso herramientas de acceso al aprendizaje del lenguaje y las matemáticas I permitió generar mayores oportunidades de aprendizaje, ya que se utilizaron diversas estrategias para que todos los estudiantes logren los resultados de aprendizaje, desarrollen las competencias profesionales y se involucren más en el proceso formativo. 
2. Los resultados de la investigación dan cuenta que las estrategias-recursos pedagógicos utilizados en el curso para la implementación del DUA contribuyeron al aprendizaje y participación de los estudiantes, siendo el material didáctico manipulativo, el material preparatorio y el material audiovisual los más valorados por los estudiantes.

3. Es necesario que en próximos cursos se fortalezcan las estrategias digitales, ya que presentan características asociadas a la flexibilidad, que hacen que sean pertinentes para la metodología DUA. En este trabajo se utilizaron foros y wiki. Sin embargo, no fueron valoradas positivamente por los estudiantes.

4. Es necesario para próximos cursos establecer criterios específicos para la elaboración de recursos pedagógicos, como es el caso de la extensión de las presentaciones de PowerPoint y el tamaño de los archivos digitales, ya que en este estudio fueron consideraron por los estudiantes un obstaculizador para su aprendizaje.

5. Cuando se realiza una innovación en la docencia es necesario contar con condiciones mínimas en la infraestructura y organización de las instituciones. En este estudio se identificó como obstaculizadores el tamaño de las salas, la acústica y la extensión de las horas de clases. Somos conscientes que estos obstaculizadores no se relacionan de manera directa con la implementación del DUA en el curso, sin embargo, constituyen para los estudiantes una barrera para acceder al aprendizaje.

6. Desde el punto de vista del diseño del estudio (investigación-acción) hay que señalar que la implementación del DUA en el curso herramientas de acceso al aprendizaje del lenguaje y las matemáticas I ha nutrido las prácticas pedagógicas de las profesoras.

\section{AGRADECIMIENTOS:}

Los autores agradecen el financiamiento de la Agencia Nacional de Investigación y Desarrollo (ANID) / Beca de Doctorado Nacional 21170975.

\section{REFERENCIAS}

Alba, C., Zubillaga, A. y Sánchez, J., Tecnologías y diseño universal para el aprendizaje (DUA): experiencias en el contexto universitario e implicaciones en la formación del profesorado, doi: https://doi.org/10.17398/1695-288X.14.1.89, Revista Latinoamericana de Tecnología Educativa, 14(1), 89-100 (2015).

Alvarado, I., Vega, Z. y otros dos autores, Comparación de estrategias de estudio y autorregulación en universitarios, Revista Electrónica de Investigación Educativa, 16(1), 137-148 (2014).

Consejo Nacional de Educación, Matrícula total de educación superior años 2005-2019, Santiago, Chile (2020).

Coy, K., Marino, M. T. y Serianni, B., Using universal design for learning in synchronous online instruction, doi: https://doi.org/10.1177/016264341402900105, Journal of Special Education Technology, 29(1), 63-74 (2014).

Dean, T., Lee-Post, A. y Hapke, H., Universal design for learning in teaching large lecture classes, doi: https://doi.org/10.1177\%2F0273475316662104, Journal of Marketing Education, 39(1), 5-16 (2016).

Díez, E. y Sánchez, S., Diseño universal para el aprendizaje como metodología docente para atender a la diversidad en la universidad, doi: https://doi.org/10.1016/j.aula.2014.12.002, Aula Abierta, 43(2), 87-93 (2015).

Edyburn, D. L., Would you recognize universal design for learning if you saw it? ten propositions for new directions for the second decade of UDL, doi: https://doi.org/10.1177/073194871003300103, Learning Disability Quarterly, 33, 33-41 (2010).

Gutiérrez-Saldivia, X., Díaz-Levicoy, D. y otros dos autores, Acceso a la información en el libro de texto de matemática de primer año en educación primaria en Chile. Espacios, 40(41), 1-22 (2019).

Inzunza, J., Assael, J. y otros dos autores, Public education and student movements: the chilean rebellion under a neoliberal experiment, doi: https://doi.org/10.1080/01425692.2019.1590179, British Journal of Sociology of Education, 40(4), 490-506 (2019).

Katz, J., Implementing the three block model of universal design for learning: effects on teachers' self-efficacy, stress, and job satisfaction in inclusive classrooms K-12, doi: 10.1080/13603116.2014.881569, International Journal of Inclusive Education, 19(1), 1-20 (2015).

King-Sears, M., Universal design for learning: technology and pedagogy, doi: https://doi.org/10.2307/27740372, Learning Disabilities Quarterly, 32(4), 199-201 (2009).

Kortering, L. J., McLannon, T. W. y Braziel, M., Universal design for learning: a Look at what algebra and biology students with and without high incidence conditions are saying, doi: https://doi.org/10.1177/0741932507314020, Remedial and Special Education, 29(6), 352-363 (2008). 
Labra, P., Montenegro, G. y otros dos autores, La investigación-acción como herramienta para lograr coherencia de acción en el proceso de práctica profesional durante la formación inicial docente, doi: https://dx.doi.org/10.4067/S071807052005000200009, Estudios Pedagógicos, 31(2), 137-143 (2005).

Mangiatordi, A. y Serenelli, F. Universal design for learning: a meta-analytic review of 80 abstracts from peer reviewed journals, Research on Education and Media, 5(1), 109-118 (2013).

Meyer, A., Rose, D. y Gordon, D., Universal design for learning: theory and practice, 1르. Ed., 1-238, CAST, Wakefield, MA, United States (2014).

Ministerio de Educación, Decreto № 83: aprueba criterios y orientaciones de adecuación curricular para estudiantes con necesidades educativas especiales de educación parvularia y educación básica, Santiago, Chile (2015).

Moore, E. J., Smith, F. G. y otros dos autores, Voices from the field: implementing and scaling-up universal design for learning in teacher preparation programs, doi: https://doi.org/10.1177/0162643417732293, Journal of Special Education Technology, 33(1), 40-53 (2018).

Parker, D., Robinson, L. y Hannafin, R., Blending technology and effective pedagogy in a core course for preservice teachers, Journal of Computing in Teacher Education, 24, 49-54 (2008).

Rao, K., Wook, M. y Bryant, B., A review of research on universal design educational models, doi:10.1177/0741932513518980, Remedial and Special Education, 35(3), 153-166 (2014).

Rao, K. y Tanners, A., Curb cuts in cyberspace: universal instructional design for online courses, Journal of Postsecondary Education and Disability, 24(3), 211-229 (2011).

Roberts, K., Park, H. y otros dos autores, Universal design for instruction in postsecondary education: a systematic review of empirically based articles, Journal of Postsecondary Education and Disability, 24(1), 5-15 (2011).

Rose, D. H., Harbour, W. y otros cuatro autores, Universal design for learning in postsecondary education: reflections and principles and their application, Journal of Postsecondary education and disability, 19(2), 135-151 (2006).

Rose, D. H. y Strangman, N., Universal design for learning: meeting the challenge of individual learning differences through a neurocognitive perspective, doi: https://doi.org/10.1007/s10209-006-0062-8, Universal Access in the Information Society, 5, 381-391 (2007).

Sánchez-Gómez, V. y López, M., Comprendiendo el diseño universal desde el paradigma de apoyos: DUA como un sistema de apoyos para el aprendizaje, doi: http://dx.doi.org/10.4067/S0718-73782020000100143, Revista Latinoamericana de Educación Inclusiva, 14(1), 143-160 (2020).

Solís-Espallargas, C. y Barreto-Tovar, C., La visión de la educación ambiental de estudiantes de maestría en pedagogía en el marco de la cátedra de la Paz en Colombia, doi: http://dx.doi.org/10.4067/S0718-50062020000200153, Formación Universitaria, 13(2), 153-166 (2020).

Undurraga, T., Neoliberalism in Argentina and Chile: common antecedents, divergent paths, doi: https://doi.org/10.1590/1678-987315235502, Revista de Sociologia e Política, 23(55), 11-34 (2015).

UNESCO, Inclusión a la universidad de estudiantes meritorios en situación de vulnerabilidad social. 1르. Ed., UNESCO, Santiago, Chile (2015).

Villarroel, V. y Bruna, D., Competencias pedagógicas que caracterizan a un docente universitario de excelencia: un estudio de caso que incorpora la perspectiva de docentes y estudiantes, doi: http://dx.doi.org/10.4067/S071850062017000400008, Formación Universitaria, 10(4), 75-96 (2017).

Yuval, L., E. Procter, K. y otros dos autores, Evaluation report on the universal instructional design at the University of Guelph, University of Guelph, Guelph, Canada (2004). 